\title{
Reliability prediction of the integrated transmission based on interval AHP and safety factor
}

\author{
Du Minggang ${ }^{1, a}$, Sun Xuan ${ }^{2, b}$, Zhang Jianguo ${ }^{2}$ and Chen Juan ${ }^{1}$ \\ ${ }^{1}$ Science and technology on vehicle transmission lab, China north vehicle institute, Beijing, China \\ ${ }^{2}$ School of Reliability and Systems Engineering, Beihang University, Beijing, China \\ a120108980@qq.com, bunxuan1027@163.com
}

Keywords: interval AHP; safety factor; reliability prediction

\begin{abstract}
In view of the characters that specimens of the integrated transmission are not sufficient, the failure modes are various and available data are not enough, this paper proposes a method utilizing experts' experiences, similar products and typical design parameters to predict reliability. First, analyze the relationship between the whole system and its components, the reliability prediction model is built accordingly. relative data of similar products are used to predict the reliability of standard and commonly used components. An evaluation model was built and the reliability correction factor was obtained via interval AHP so as to realize the mutual use of certain and fuzzy information. For uncommonly used components, the safety factor is employed to predict its reliability. Finally, the application of this method is illustrated by case of integrated transmission.
\end{abstract}

\section{Introduction}

Reliability prediction is of significant importance in the reliability design process of integrated transmission. It can verify whether the requirements in the assignment book are achieved, compare and choose the best design project from aspects of both function and reliability, and find out the week points to make some improvement ${ }^{[1,2,3,4]}$.

Researches about reliability prediction are all begin with electronics industry. Now there are a lot of standards and manuals in the electronic industry because of the strong university and high level of standardization ${ }^{[5]}$. However, mechanical parts are much more sensitive to loads and working environment, which leads to be less universe or standard ${ }^{[6]}$.

Now reliability prediction methods applied in typical mechanical products include similar product method, experts scoring method and so on $^{[6]}$.However in view of the problem that mechanical components have various failure modes, complex loading situations, small number of specimen and many ignored design parameters, a reliability prediction method realizing mutual use of similar products and safety factor was presented. This method takes advantages of abundant experience of experts, the uncertain information during its lifetime and relative design parameters, tending to be much more realistic and precise.

\section{The Reliability Prediction Model of Integrated Transmission}

The flow chart of integrated transmission's reliability prediction is showed in Figure 1.

\section{Build the Reliability Block Diagram}

Integrated transmission consists of twenty parts in series such as the overall drive device, body parts, and so on, which are denoted by $S_{1}, S_{2}, \ldots, S_{20}$. Then the reliability block diagram can be built accordingly in Fig. 2.

\section{Build the Reliability Prediction Model of Integrated Transmission}

The relationship between the reliability of integrated transmission and its subsystems is as follow:

$$
R_{S}(t)=R_{1}(t) \cdot R_{2}(t) \cdots R_{20}(t)
$$


Where $R_{S}(t)$ represents the reliability of the integrated transmission (system), $R_{i}(t)(1 \leq i \leq 20)$ is the reliability of $i$ th subsystem.

For $R(t)=e^{-\int_{0}^{t} \lambda(t) d t}$, combined with formula (1), then

$$
R_{S}(t)=e^{-\int_{0}^{t} \lambda_{S}(t) d t}=\prod_{i=1}^{20} e^{-\int_{0}^{t} \lambda_{i}(t) d t}=\prod_{i=1}^{20} R_{i}(t)
$$

Where $\lambda_{s}(t)$ is the failure rate of system, $\lambda_{i}(t)$ is the failure rate of $i$ th subsystem, formula(2) turns to:

$$
\lambda_{S}(t)=\sum_{i=1}^{20} \lambda_{i}(t)
$$

Then, the failure rate of system equals to the sum of that of every subsystems.

There are some difficulty in reliability prediction in engineering with formula (1)and (3) since the failure time of different subsystems may not follow the same distribution. Mean failure rate can solve this problem by replacing the failure rate. It can be defined as follows: when $t=T$, namely the ending time of the test is $T$, the mean failure rate of product between $[0, T]$ is $\bar{\lambda}$.

$$
\bar{\lambda}=\frac{1}{T} \int_{0}^{T} \lambda(t) d t
$$

Substitute formula (4) into (3), it turns out:

$$
\bar{\lambda}_{s}=\bar{\lambda}_{1}+\bar{\lambda}_{2}+\ldots+\bar{\lambda}_{20}
$$

Where $\bar{\lambda}_{s}$ is the mean failure rate of system and $\bar{\lambda}_{i}$ is that of $i$ th subsystem, $1 \leq i \leq 20$.

So the whole system's reliability can be obtained by the model mentioned above.

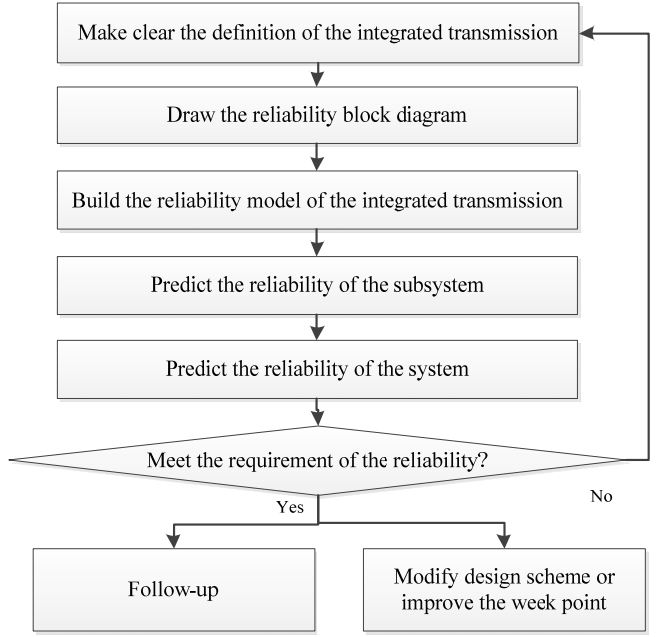

Fig 1 the flow chart of the prediction process

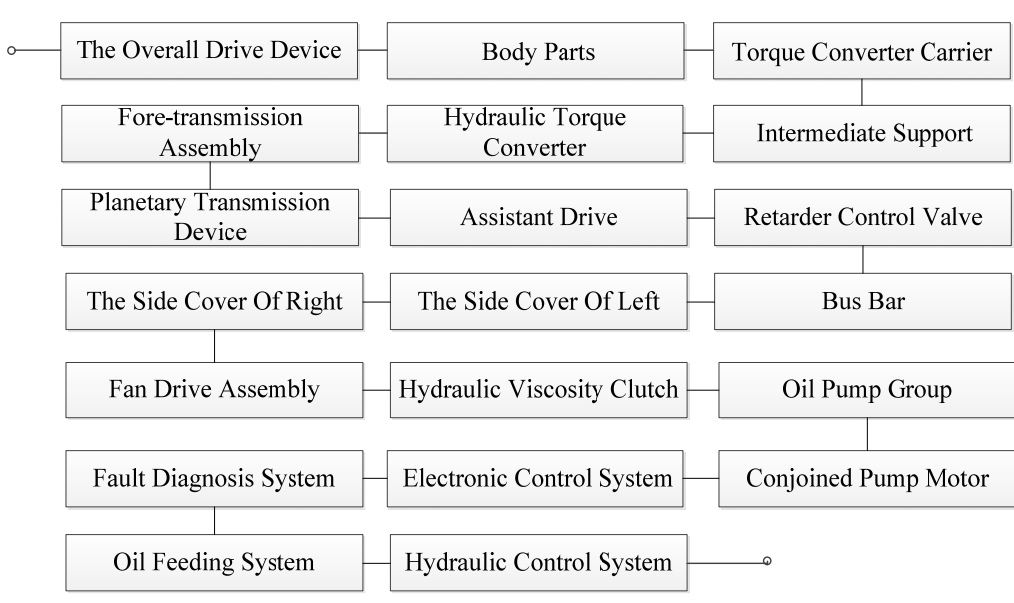

Fig 2 the reliability block diagram of integrated transmission

\section{The Reliability Prediction Model of Every Component}

There are two kinds of subsystems in the integrated transmission, widely used one and independently designed one. The former's reliability is predicted via the method of similar products, while the later makes use of safety factor to carry on the reliability prediction.

\section{The Method of Similar Products}

Take the subsystem $S_{i}$ as example, assuming that $m$ similar products were chosen. Among them, the mean failure rate of $j$ th similar subsystem is $\bar{\lambda}_{i j}(1 \leq j \leq m)$.

Taking consideration of the differences between the research object and the similar products, the reliability correction factor was induced. Therefore the mean failure rate of the subsystem $S_{i}$ is: 


$$
\bar{\lambda}_{i}=\frac{1}{m} \sum_{j=1}^{m}\left(\bar{\lambda}_{i j} / W_{j}\right)
$$

Where $W_{j}$ is the reliability correction factor of the $j$ th similar product.

\section{The Evaluating Model of the Reliability Correction Factor.}

The qualification relationship between $W_{j}$ and its influence factors is built as follows. By looking up to relative handbooks and analyzing large amount of field failure data, the comprehensive and hierarchical evaluation model of the reliability correction is built in Fig.3.

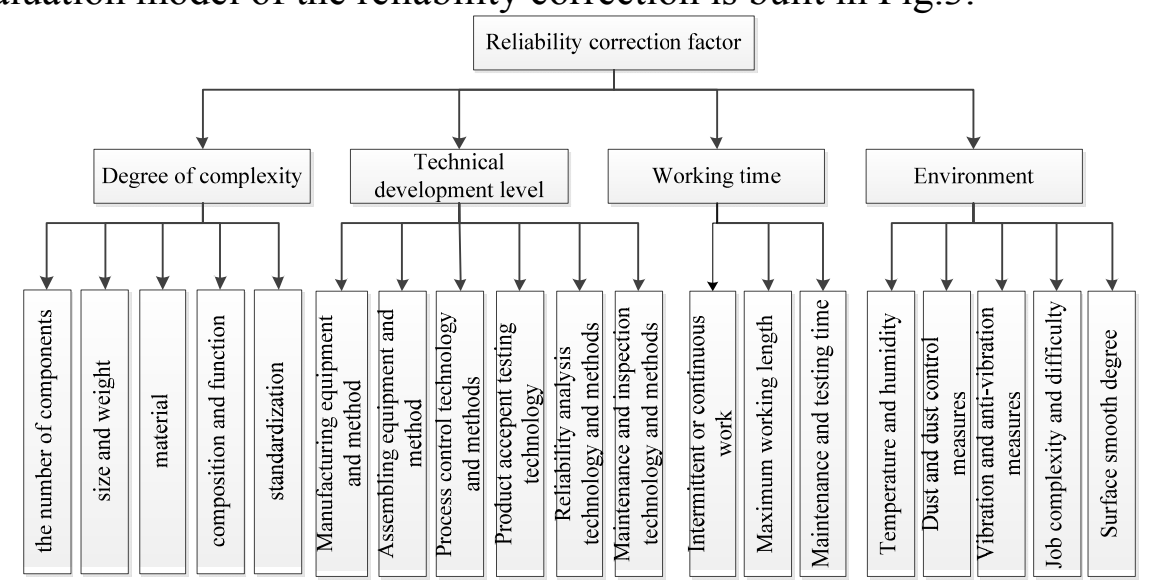

Fig 3 the comprehensive and hierarchical evaluation model of the reliability correction

There are three layers in this hierarchical evaluation model, object layer on the top, criterion layer in the middle and the index layer in the bottom. The four categories in the criterion layer are represented by $U_{1}, U_{2}, U_{3}, U_{4}$ and then the set of them is $U=\left\{U_{1}, U_{2}, U_{3}, U_{4}\right\}$. Take "Degree of complexity" as example, the sub-factors include five index, representing by $u_{11}, u_{12}, \cdots, u_{15}$

\section{The Weight of Categories.}

Generally, three scales, five scales or nine scales are used to measure the judging matrix in AHP(Analytic hierarchy process). But the fuzziness of experts' judgment is ignored ${ }^{[7,8]}$. Therefore the four categories were weighed by interval number $\tilde{a}_{k l}=\left[a_{k l}^{-}, a_{k l}^{+}\right]$according to experts' advices and following the scale from one to nine. Then every category's judging matrix $\AA$ can be obtained.

$$
A=\left(\tilde{a}_{k l}\right)_{4 \times 4}=\left[A^{-}, A^{+}\right]
$$

Where $\tilde{a}_{k l}=\left[a_{k l}^{-}, a_{k l}^{+}\right]$denotes the ratio of the $k$ th and $l$ th category for the object, $1 \leq k \leq 4,1 \leq l \leq 4$; $A^{-}=\left(a_{k l}^{-}\right)_{4 \times 4}, A^{+}=\left(a_{k l}^{+}\right)_{4 \times 4}$.

As $\tilde{a}_{k l}=1 / \tilde{a}_{l k}$, only the upper triangular element values of the matrix is necessary. The weight vector of each category, which represents relative importance degree, is obtained by interval eigenvector method (IEM) and the calculate process is as follows: the weight vector of $A^{-}, A^{+}$can be obtained via eigenvalue vector method and marked as $x^{-}, x^{+}$.Then the interval weight vector is:

$$
\begin{aligned}
& \varpi=\left(\omega_{k}\right)_{1 \times 4}=\left[\alpha x^{-}, \beta x^{+}\right] \\
& \alpha=\left[\sum_{l=1}^{4}\left(\sum_{k=1}^{4} a_{k l}^{+}\right)^{-1}\right]^{\frac{1}{2}}, \beta=\left[\sum_{l=1}^{4}\left(\sum_{k=1}^{4} a_{k l}^{-}\right)^{-1}\right]^{\frac{1}{2}}
\end{aligned}
$$

If $A^{-}=A^{+}$, the weight vector from the IEM mentioned above equals to that from eigenvalue method. 


\section{The Weight of Sub-Factors and Their Differences.}

The importance degree of every sub-factor is determined by the same way as that of categories. The weight vectors of every sub-factor is:

$$
\varpi_{k}=\left(\omega_{k v}\right)_{1 \times n}=\left[\alpha_{k} x_{k}^{-}, \beta_{k} x_{k}^{+}\right]
$$

Where $1 \leq k \leq 4$ and $\varpi_{k v}$ is the weight of $v$ th sub-factors belonging to the $k$ th category.

Compare every sub-factor in the index layer, then the difference of $v$ th sub-factor belonging to the $k$ th category is denoted by $Y_{k v}=\left[Y_{k v}^{-}, Y_{k v}^{+}\right]$, and it equals to [1,1] if they are the same. If the changing in the new product improves its reliability, $\mathrm{Y}_{k v}$ is interval number from one to nine otherwise is the reciprocal of the interval numbers.

The Differences between Categories.

Calculate the difference of $k$ th category $B_{k}$ according to the weight of sub-factor $\varpi_{k}$ and the difference between sub-factors $Y_{k v}$.

$$
B_{k}=\sum_{v=1}^{n}\left(\omega_{k v} \cdot Y_{k v}\right)
$$

\section{Calculating the Reliability Correction Factor.}

With weight $\mathscr{\omega}_{k}$ and the difference of sub-factors $\Psi_{k v}$, reliability correction factor $W_{j}$ can be obtained.

$$
W_{j}=\sum_{k=1}^{4}\left(B_{k} \cdot \mathscr{\omega}_{k}\right) / \sum_{j=1}^{m} \sum_{k=1}^{4}\left(B_{k} \cdot \omega_{k}\right)
$$

A decision factor $p$ is induced to do the defuzzification work and the specific method is as follows:

$$
W_{j}=W_{j}^{-}+p\left(W_{j}^{+}-W_{j}^{-}\right)
$$

Where the decision factor $p 0 \leq p \leq 1$ is determined by experts.

Substitute $W_{j}$ and $\lambda_{i j}$ into formula (6) and the mean failure rate of $i$ th subsystem is obtained.

\section{The Method of Safety Factor.}

The method of structural strength safety factor is widely used in structure design because of its simplicity ${ }^{[10]}$. Many components such as spindles, gears and splines can take this design method, which will lead to a large number of parameters available for reliability prediction.

Tolerable Safety Factor.

It is showed in relevant rules of JSSG-2006 that the tolerable value of design benchmark should be used in analyzing structural strength. Numerical grades, definition and suitable object can refer to MIL-HDBK-5C ${ }^{[11]}$.

When strength and stress both obey normal distribution, the structural tolerable safety factor can be expressed as the following formula.

$$
n(r, \gamma)=n_{m}\left[1-k(r, \gamma) C_{r}\right]
$$

Where $k(r, \gamma)$ is the lower limit of tolerance coefficient with reliability $r$, confidence $\gamma$ and normal distribution. $n(r, \gamma)$ denotes the structural tolerant safety factor; $n_{m}$ denotes the center safety coefficient; $C_{r}$ denotes the variation coefficient of strength.

\section{The Relationship between Tolerable Safety Factor and Reliability}

Basis assumptions ${ }^{[11]}$ :

- The performance parameters of material's static strength obey normal distribution.

- The load of structure in using process obeys normal distribution. 
- The load and the performance parameters of material are statistically independent.

The relationship between the reliability of structural static strength and tolerable safety factor can refer to literature ${ }^{[11]}$.

$$
\begin{aligned}
& \beta_{R}=\frac{n(r, \gamma)+k(r, \gamma) C_{r}-1}{\sqrt{\left[C_{s}-k(r, \gamma) C_{s} C_{r}\right]^{2}+\left[n(r, \gamma) C_{s}\right]^{2}}} \\
& R=\Phi\left(\beta_{R}\right)
\end{aligned}
$$

Where $\beta_{R}$ denotes the index of reliability. $C_{s}$ and $C_{r}$ represent the variation coefficient of stress and strength respectively.

\section{Reliability Prediction Based on the Tolerable Safety Factor}

Take the main spindle of integrated transmission as example and substitute the design basis, the tolerable safety factor and material parameters into formula (15),(16). Then the reliability of the spindle is predicted.

\section{Case study}

Take the integrated transmission of a heavy vehicle as an example and predict its reliability by the methods presented in this paper.

\section{The Method of Similar Products}

The AHP method is applied to the reliability prediction of the front drive assemble. Three similar products were chosen with mean failure rates $\bar{\lambda}_{21}=2.482 \times 10^{-5}, \bar{\lambda}_{22}=2.306 \times 10^{-5}$ and $\bar{\lambda}_{23}=2.592 \times 10^{-5}$, and the corresponding mean kilometers before failure(MKBF) are $M K B F_{21}=40290, M K B F_{22}=43365, M K B F_{23}=38580$, respectively. For the four categories in criterion layer, several experts were chosen to make a score according to the judging principles and an interval judging matrix $A$ can be obtained from table1.

Table 1the weight matrix of categories in criterion layer

\begin{tabular}{|c|c|c|c|c|}
\hline & $U_{1}$ & $U_{2}$ & $U_{3}$ & $U_{4}$ \\
\hline$U_{1}$ & {$[1,1]$} & {$[0.2,0.4]$} & {$[0.4,0.6]$} & {$[0.1,0.7]$} \\
\hline$U_{2}$ & & {$[1,1]$} & {$[0.3,0.5]$} & {$[0.2,0.7]$} \\
\hline$U_{3}$ & & & {$[1,1]$} & {$[0.3,0.4]$} \\
\hline$U_{4}$ & & & & {$[1,1]$} \\
\hline
\end{tabular}

The weight of every category is computed as follows according to IEM.

$$
\varpi=[0.1390,0.2711],[0.3180,0.3472],[0.1816,0.1905],[0.1972,0.3390]
$$

After obtaining the weight of every category, the weight and differences between the sub-factors are to calculate. The weight matrix of "complexity" is founded firstly.

Table 2the weight matrix of "complexity"

\begin{tabular}{|c|c|c|c|c|c|}
\hline & $u_{11}$ & $u_{12}$ & $u_{13}$ & $u_{14}$ & $u_{15}$ \\
\hline$u_{11}$ & {$[1,1]$} & {$[1.2,1.9]$} & {$[1.4,1.8]$} & $[0.9,2.1]]$ & {$[0.9,1.7]$} \\
\hline$u_{12}$ & & {$[1,1]$} & {$[0.7,1.6]$} & {$[0.2,0.5]$} & {$[0.2,0.7]$} \\
\hline$u_{13}$ & & & {$[1,1]$} & {$[0.3,0.9]$} & {$[0.2,0.7]$} \\
\hline$u_{14}$ & & & & {$[1,1]$} & {$[0.9,1.5]$} \\
\hline$u_{15}$ & & & & & {$[1,1]$} \\
\hline
\end{tabular}

The weight of every sub-factor is computed as follows according to IEM. 


$$
\mathscr{\omega}_{\omega}=[0.1551,0.2790],[0.0694,0.1365],[0.0793,0.1269],[0.1896,0.2360],[0.1620,0.2697]
$$

Compare the design product with the first similar product and the difference between the five sub-factors in complexity is obtained in table3.

Table 3 the difference of the 5 sub-factors of complexity

\begin{tabular}{|c|c|c|c|c|c|}
\hline Sub-factor & $u_{11}$ & $u_{12}$ & $u_{13}$ & $u_{14}$ & $u_{15}$ \\
\hline Degree of difference & {$[0.9,1.3]$} & {$[1,1.4]$} & {$[0.7,1.3]$} & {$[0.8,1.2]$} & {$[0.7,0.9]$} \\
\hline
\end{tabular}

Substituting the difference and weight into formula(11), the difference of complexity can be obtained $B_{1}=[0.52958,1.2447]$. Then the differences of the other three categories can be obtained in the similar way which has been showed in table4.

Table 4the difference degree of categories

\begin{tabular}{|c|c|c|c|c|}
\hline categories & $U_{1}$ & $U_{2}$ & $U_{3}$ & $U_{4}$ \\
\hline Degree of difference & {$[0.52958,1.2447]$} & {$[0.8659,0.9781]$} & {$[0.6291,1.1583]$} & {$[1.0536,1.3421]$} \\
\hline
\end{tabular}

With the result above, the reliability correction factor of the first similar product is $W_{1}=[0.584,1.106]$. According to the experts' experiences, let $p=0.45$ conservatively, then $W_{1}=0.8189$ which shows that the reliability is slightly lower than the first similar product.

Similarly, the reliability correction factor of the other two similar products is $W_{2}=1.251$, $W_{3}=1.062$ and the mean failure rate of the front drive assemble can be obtained by substituting them into formula(6) $\bar{\lambda}_{2}=2.438 \times 10^{-5}$, and the corresponding $M K B F_{2}=40107$.

\section{The Method Based on Safety Factor}

Taking the main spindle of the integrated transmission as example, the method based on the safety factor is used to predict its reliability.

"A" basis design criteria is adopted according to the design requirements, and $k(r, \gamma)=k(0.99,0.95)=2.684$. The material of the main spindle is 40CrNi2Si2MoVA, whose performance parameters are as follows in table5.

Table 5 performance parameters of material

\begin{tabular}{|c|c|c|c|c|}
\hline$A[\mathrm{MPa}]$ & $\bar{r}[\mathrm{MPa}]$ & $s_{r}[\mathrm{MPa}]$ & $C_{r}$ & $n$ \\
\hline 380.3 & 456.6 & 28.2 & 0.062 & 100 \\
\hline
\end{tabular}

The structural stress variation coefficient is $C_{s}=0.12$ conservatively taking the uncertainty of the expected load of the main spindle.

$$
n(0.99,0.95)=1.3 \text { referring to the literature[11]. }
$$

Substituting the parameters above into formula(15) $\beta_{R}=3.63$ and then the reliability $R=0.999858$.

Similarly, the mean failure rate of other subsystems of the integrated transmission can be computed and substitute them into formula(5) or (1), the reliability of the whole system can be obtained.

\section{Summary}

On the basis of similar product prediction method, a reliability prediction method based on interval hierarchy analysis is presented in this paper, which compares the aspects of similar products such as complexity, technical level, and working time. By inducing the reliability correction factor, the reliability prediction model of system and subsystems is built, which realizes the comprehensive utilization of both certain and uncertain information and also provides a solution to predict reliability under the circumstance of data lacking. 
A reliability prediction method for structural components based on the safety factor is presented, which can make use of the design parameters, material parameters as well as relative experts' experiences and leading a more realistic result.

The applied case verifies the feasibility and practice meaning of the method presented in the engineering.

\section{References}

[1] Blischke W R and Murthy D N P. Reliability: Modeling, Prediction, and Optimization. New York. Wiley, (2000), p517-519.

[2] MIL-HDBK-217F,Notice 2.Military handbook "reliability prediction of electronic equipment". Washington DC, Department of Defence, (1995).

[3] NSMC-06.Handbook of reliability prediction procedures for mechanical equipment.Maryland: Naval Surface Warfare Center, Department of Defence,(2006).

[4] Zeng Shengkui.Reliability Design and Analysis.Beijing: National Defense Industry Press, (2011) p14-21.

[5] Zhang Wenjun, Nie Guojian and Zheng Lixiang. Overview of the Latest Reliability Prediction Method. Electronic Reliability and Environmental Testing, vol.27, (2009) p21-28,

[6] Li Ruiying, Kang Rui and Dang Wei. Comparison and Selection of Prediction Method for Mechanical Products Reliability. Engineering Machinery. vol.40:( 2009).p53-57.

[7] Hao Qingbo.Fuzzy Comprehensive Allocation and Prediction for NC Machine Tools Reliability and Maintainability.Jinlin: the major of Mechanical Manufacture and Automation in Jinlin University(2012).

[8] Hao Qingbo, Yang Zhaojun and Chen Chuanhai. Reliability Prediction for NC Machine Tool based on interval AHP, Journal of Jinlin University (Engineering and Technology Edition),vol.24:(2012).p845-850 .

[9] Moore R, Lodwick W. Interval analysis and fuzzy set theory. Fuzzy Sets and Systems,vol.135, (2003)p.5-9.

[10] Wang Lei, Liu Wenting. Method of reliability design for aircraft structural ststic strength based on tolerable safety factor, Journal of Mechanical Strength, vol.31:(2009) p 487- 490.

[11] Liu Wenting. Handbook of structural reliability design.Beijing: National Defense Industry Press, (2008),p478-486. 